\title{
Can quantum chaos enhance stability of quantum computation?
}

\author{
Tomaž Prosen and Marko Žnidarič \\ Physics Department, Faculty of Mathematics and Physics, University of Ljubljana, Slovenia
}

(October 27, 2018)

\begin{abstract}
We consider stability of a general quantum algorithm with respect to a fixed but unknown residual interaction between qubits, and show a surprising fact, namely that the average fidelity of quantum computation increases by decreasing average time correlation function of the perturbing operator in sequences of consecutive quantum gates. Our thinking is applied to the quantum Fourier transformation where an alternative 'less regular' quantum algorithm is devised which is qualitatively more robust against static random residual $n$ qubit interaction.
\end{abstract}

PACS number: 03.67.Lx, 05.45.-a

Recent investigations of theoretical and experimental possibilities of quantum information processing have made the idea of quantum computation [1] very attractive and important (see e.g. [2] for a review). Having the apparatus which is capable of manipulation and measurement on pure states of individual quantum systems one can make use of massive intrinsic parallelism of coherent quantum time evolution.

The main idea of quantum computation is the following: Consider a many-body system of $n$ elementary twolevel quantum excitations - qubits, which is called the quantum register, store the data for quantum computation in the initial state of a register $|\mathrm{r}\rangle$ which is a superposition of an exponential number $\mathcal{N}=2^{n}$ of basic qubit states, then perform certain unitary transformation $U$ by decomposing $U=U(T) \cdots U(2) U(1)$ into a sequence of $T$ elementary one-qubit and two-qubit quantum gates $U(t)$, $t=1,2, \ldots, T$, such decomposition being called a quantum algorithm (QA), and in the end obtain the results by performing measurements of qubits on a final register state $U|\mathrm{r}\rangle$. QA is called efficient if the number of needed elementary gates $T$ grows with at most polynomial rate in $n=\log _{2} \mathcal{N}$, and only in this case it can generally be expected to outperform the best classical algorithms (in the limit $n \rightarrow \infty)$. At present only few efficient QAs are known, and perhaps the most generally useful is the Quantum Fourier transformation (QFT) [3].

There are two major obstacles for performing practical quantum computation: First, there is a problem of decoherence [4] resulting from an unavoidable time-dependent coupling between qubits and the environment. If the perturbation couples only a small number of qubits at a time then such errors can be eliminated at the expense of extra qubits by quantum error correcting codes [5] (see Ref. [6] for another approach). Second, even if one knows an efficient error correcting code or assumes that quan- tum computer is ideally decoupled from the environment, there will typically exist a small unknown or uncontrollable residual interaction among qubits which one may describe by a general static perturbation. Therefore, understanding the stability of QAs with respect to various types of perturbations is an important problem (see 79 for some results on this topic).

Motivated by [10], we propose a new approach to the stability of quantum computation with respect to a static but incurable (perhaps unknown) perturbing interaction. We consider QA as a time-dependent dynamical system and relate its fidelity measuring the Hilbert space distance between computed states of exact and perturbed algorithm in terms of integrated time-autocorrelation of the perturbing operator (generalizing Ref. [11]). The derived relation looks very surprising: it tells that faster decay of time-correlations of the perturbation between sequences of successive quantum gates means larger fidelity, and vice versa. We propose to use our rule of thumb as a guide to devise or to improve QAs, either by introducing extra 'chaotic' gates or by rewriting the gates in a different order in order to make time-evolution $U(t)$ 'more chaotic'. As an important example, the well known QFT algorithm whose internal dynamics appears unpleasantly 'regular' has been improved in a way that the modified algorithm becomes qualitatively more robust against static random perturbation of the gates. We think our effect should be considered in experimental realization of QFT which are underway [12].

Let us write the partial evolution operator for a sequence of consecutive gates from $t^{\prime}$ to $t, t^{\prime}<t$, as $U\left(t, t^{\prime}\right)=U(t) U(t-1) \cdots U\left(t^{\prime}+2\right) U\left(t^{\prime}+1\right)$, with $U(t, t) \equiv$ 1 , and perturb the quantum gates by a (generally timedependent) perturbation of strength $\delta$ generated by hermitean operators $V(t)$

$$
U_{\delta}(t)=U(t) \exp (-i \delta V(t)) .
$$

Propagating the initial register state $|\mathrm{r}\rangle$ with exact and perturbed algorithms we focus on the fidelity of the QA defined as

$$
F(T)=\frac{1}{\mathcal{N}} \operatorname{tr} U_{\delta}^{\dagger}(T, 0) U(T, 0)
$$

as an average over all initial register states. Defining the Heisenberg time evolution from $t^{\prime}$ to $t, V\left(t, t^{\prime}\right)=$ $U^{\dagger}\left(t, t^{\prime}\right) V(t) U\left(t, t^{\prime}\right)$, we rewrite the fidelity as

$$
F(T)=\frac{1}{\mathcal{N}} \operatorname{tr}\left(e^{i \delta V(1,0)} e^{i \delta V(2,0)} \cdots e^{i \delta V(T, 0)}\right)
$$

by $T$ insertions of the unity $U^{\dagger}(t, 0) U(t, 0)=1$ and observing $U^{\dagger}(t-1,0) U_{\delta}^{\dagger}(t) U(t, 0)=\exp (i \delta V(t-1,0))$. 
Next we make a series expansion in $\delta$ expressing the fidelity in terms of correlation functions

$$
F(T)=1+\frac{1}{\mathcal{N}} \sum_{m=1}^{\infty} \frac{i^{m} \delta^{m}}{m !} \sum_{t_{1}, \ldots, t_{m}=1}^{T} \operatorname{tr}\left(\hat{\mathcal{T}} \prod_{j=1}^{m} V\left(t_{j}, 0\right)\right)
$$

where $\hat{\mathcal{T}}$ is a left-to-right time ordering (w.r.t. indices $t_{j}$ ). We can make the series starting at second order $m=$ 2 by assuming the average perturbation to be traceless, $\operatorname{tr} \bar{V}=(1 / T) \sum_{t=1}^{T} \operatorname{tr} V(t, 0) \equiv 0$ (otherwise, the effect of subtracting the trace average is a simple complex rotation of fidelity). To second order in $\delta$, the fidelity can be written as

$$
F(T)=1-\frac{\delta^{2}}{2} \sum_{t, t^{\prime}=1}^{T} C\left(t, t^{\prime}\right)+\mathcal{O}\left(\delta^{3}\right),
$$

in terms of a 2-point time correlation (correlator) of the perturbation $C\left(t, t^{\prime}\right):=C\left(t^{\prime}, t\right):=$ $\operatorname{tr}\left(V\left(t^{\prime}, 0\right) V(t, 0)\right) / \mathcal{N}=\operatorname{tr}\left(V\left(t^{\prime}\right) V\left(t, t^{\prime}\right)\right) / \mathcal{N}$. The relation (5) is very interesting: it tells that the QA is more stable if the time correlations of the perturbation are smaller on average, meaning that the 'chaotic' quantum time evolution is more stable than the 'regular' one [11]. One may use this general philosophy as a guide to design QAs, or to improve the existing ones by rearranging quantum gates.

However, the behavior of the correlation function depends also on explicit time-(in)dependence of the perturbation $V(t)$. For example, if the perturbation $V(t)$ is an uncorrelated noise, as would be in the case of coupling to an ideal heath bath, then the matrix elements of $V(t)$ may be assumed to be gaussian random with variances $\left\langle V_{j k}(t) V_{l m}\left(t^{\prime}\right)\right\rangle_{\text {noise }}=(1 / \mathcal{N}) \delta_{j m} \delta_{k l} \delta_{t t^{\prime}}$. Hence one finds $\left\langle C\left(t, t^{\prime}\right)\right\rangle_{\text {noise }}=\delta_{t t^{\prime}}$, and averaging of a formula (何) yields the noise-averaged fidelity $\langle F(T)\rangle_{\text {noise }}=\exp \left(-\delta^{2} T / 2\right)$ which is independent of the $Q A U(t)$. On the other hand, for a static residual interaction $V(t) \equiv V$ one may expect slower correlation decay, depending on the 'regularity' of the evolution operator $U$, and hence faster decay of fidelity. Importantly, note that in a physical situation, where perturbation is expected to be a combination $V(t)=V_{\text {static }}+V_{\text {noise }}(t)$, the fidelity drop due to a static component is expected to dominate long-time quantum computation $T \rightarrow \infty$ over the noise component (e.g. due to decoherence), as soon as QA exhibits long time correlations of the operator $V_{\text {static }}$. Since the sequence of gates to accomplish a certain task $U$ is by no means unique, the natural question arises, how to write the QA in order to have fastest decay of time correlations with respect to a static, say gaussian random (GUE) perturbation?

We consider QFT working in a Hilbert space of dimension $\mathcal{N}=2^{n}$ with basis qubit states denoted by $|k\rangle, \quad k=0, \ldots, 2^{n}-1$. The unitary matrix $U_{\mathrm{QFT}}$ performs the following transformation on a state with expansion coefficients $x_{k}$

$$
U_{\mathrm{QFT}}\left(\sum_{k=1}^{\mathcal{N}} x_{k}|k\rangle\right)=\sum_{k=1}^{\mathcal{N}} \tilde{x}_{k}|k\rangle
$$

where $\tilde{x}_{k}=\frac{1}{\sqrt{\mathcal{N}}} \sum_{j=1}^{\mathcal{N}} \exp (2 \pi i j k / \mathcal{N}) x_{j}$. "Dynamics" of QFT consists of three kinds of unitary gates: One-qubit gates $\mathrm{A}_{j}$ acting on $j$-th qubit

$$
\mathrm{A}_{j}=\frac{1}{\sqrt{2}}\left(\begin{array}{cc}
1 & 1 \\
1 & -1
\end{array}\right)
$$

diagonal two-qubit gates $\mathrm{B}_{j k}=\operatorname{diag}\left\{1,1,1, \exp \left(i \theta_{j k}\right)\right\}$, with $\theta_{j k}=\pi / 2^{k-j}$, and transposition gates $\mathrm{T}_{j k}$ which interchange $j$-th and $k$-th qubits. There are $n$ A-gates, $n(n-1) / 2 \mathrm{~B}$-gates and $[n / 2]$ transposition gates, where $[x]$ is an integer part of $x$. The total number of gates for the whole algorithm is therefore $T=[n(n+2) / 2]$. For instance, in the case of $n=4$ we have a sequence of $T=12$ gates (time runs from right to left)

$$
U_{\mathrm{QFT}}=\mathrm{T}_{03} \mathrm{~T}_{12} \mathrm{~A}_{0} \mathrm{~B}_{01} \mathrm{~B}_{02} \mathrm{~B}_{03} \mathrm{~A}_{1} \mathrm{~B}_{12} \mathrm{~B}_{13} \mathrm{~A}_{2} \mathrm{~B}_{23} \mathrm{~A}_{3} .
$$

In what follows we will focus on a static random perturbation, that is $V(t) \equiv V$ is a random $\mathcal{N}$ dimensional GUE matrix with normalized second moments $\left\langle V_{j k} V_{l m}\right\rangle=\delta_{j m} \delta_{k l} / \mathcal{N}$, where $\langle$.$\rangle denotes an av-$ erage over GUE. For small perturbation strength $\delta$ the quantity controlling the fidelity (5) is the correlator

$$
\left\langle C\left(t, t^{\prime}\right)\right\rangle=\frac{1}{\mathcal{N}}\left\langle\operatorname{tr}\left(V\left(t, t^{\prime}\right) V\right)\right\rangle=\left|\frac{1}{\mathcal{N}} \operatorname{tr} U\left(t, t^{\prime}\right)\right|^{2} .
$$

Averaging over GUE is done only to ease up analytical calculation and to yield a quantity that is independent of a particular realization of perturbation. Qualitatively similar (numerical) results are obtained without the averaging. We have $\langle C(t, t)\rangle \equiv 1$ due to normalization of the second moments of GUE, while for an arbitrary fixed $V$, the diagonal correlator is

$$
C(t, t)=C(0,0)=\frac{1}{\mathcal{N}} \operatorname{tr} V^{2} .
$$

In a sum of correlation function (5) we must therefore distinguish two contributions: (i) The diagonal correlator (10) just sets an overall scale. This is a static quantity as it depends on the strength of a perturbation $V$ only and can be included in $\delta$ by normalizing $\operatorname{tr} V^{2} / \mathcal{N}=1$. (ii) The off-diagonal contribution is mainly determined by the rate of decay of $C\left(t, t^{\prime}\right)$ as $t-t^{\prime}$ increases which is an essential dynamical feature of QA.

We have calculated the correlator $\left\langle C\left(t, t^{\prime}\right)\right\rangle$ for QFT (9) which is shown in top fig.11. One can clearly see square red plateaus on the diagonal due to blocks of successive B-gates. Similar square plateaus can also be seen off diagonal (from orange, yellow to green), so that the correlation function has a staircase-like structure, with the A-gates responsible for the drops and B-gates responsible for the flat regions in between. This can be easily understood. For "distant" qubits $k-j \gg 1$ the gates $\mathrm{B}_{j k}$ 
are close to the identity and therefore cannot reduce the correlator. This slow correlation decay results in the correlation sum $\chi:=\frac{1}{2} \sum_{t, t^{\prime}=1}^{T} C\left(t, t^{\prime}\right)$ being proportional to $\chi \propto n^{3}$ (sum of the first $n$ squares) as compared to the theoretical minimum $\chi \propto T \propto n^{2}$.
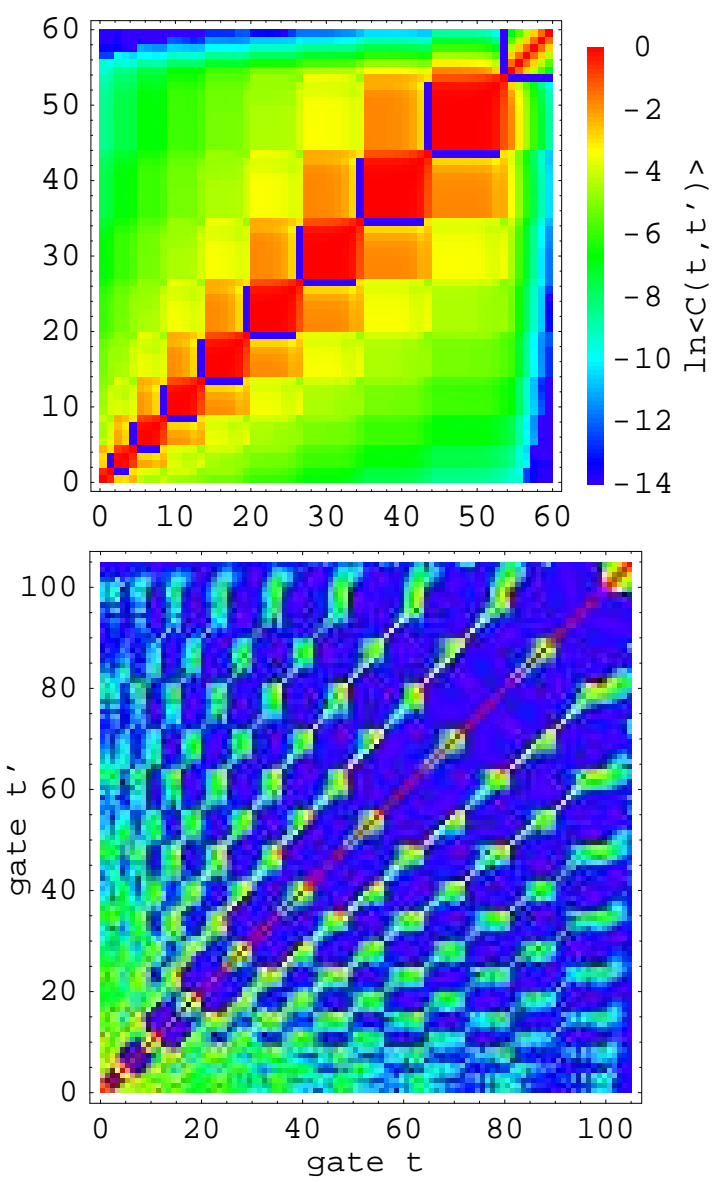

FIG. 1. Correlation function $\left\langle C\left(t, t^{\prime}\right)\right\rangle$ for $n=10$ qubits and GUE perturbation. Top figure shows standard QFT (8) with $T=60$, while bottom figure shows IQFT with $T=105$ gates. Color represents the size of elements in log-scale from red $\left(e^{-0}\right)$ to blue $\left(e^{-14}\right.$ and less).

In view of this, we will now try to rewrite the QFT with a goal to accomplish $\chi \propto n^{2}$. From (9) we learn that the gates that are traceless (e.g. A-gates) reduce the correlator very effectively. In the plain QFT algorithm (8) we have $n-1$ blocks of B-gates, where in each block all B-gates act on the same first qubit, say $j$. In each such block, we propose to replace $\mathrm{B}_{j k}$ with a new gate $\mathrm{G}_{j k}=$ $\mathrm{R}_{j k}^{\dagger} \mathrm{B}_{j k}$, where unitary gate $\mathrm{R}_{j k}$ will be chosen so as to commute with all the diagonal gates $\mathrm{B}_{j l}$ in the block, whereas at the end of the block we will insert $\mathrm{R}_{j k}$ in order to "annihilate" $\mathrm{R}_{j k}^{\dagger}$ so as to preserve the evolution matrix of a whole block. Unitarity condition $\mathrm{R}_{j k}^{\dagger} \mathrm{R}_{j k}=1$ and $\left[\mathrm{R}_{j k}, \mathrm{~B}_{j l}\right]=0$ for all $j, k, l$ leave us with a 6 parametric set of matrices $\mathrm{R}_{j k}$. By further enforcing $\operatorname{tr} \mathrm{R}_{j k}=0$ in order to maximally reduce the correlator, we end up with 4 free real parameters in $\mathrm{R}_{j k}$. One of the simplest choices, that has been proved to be equally suitable as any other, is the following

$$
\mathrm{R}_{j k}=\left(\begin{array}{cccc}
0 & 0 & -1 & 0 \\
0 & 1 & 0 & 0 \\
1 & 0 & 0 & 0 \\
0 & 0 & 0 & -1
\end{array}\right)
$$

Furthermore, we find that R-gates also commute among themselves, $\left[\mathrm{R}_{j k}, \mathrm{R}_{j l}\right]=0$, which enables us to write a sequence of R-gates as we like, e.g. in the same order as a sequence of G's, so that pairs of gates $\mathrm{G}_{j k}, \mathrm{R}_{j k}$ operating on same pair of qubits $(j, k)$, whose product is a bad gate $\mathrm{B}_{j k}$, are never neighboring. This is best illustrated by an example. For instance, the block $\mathrm{B}_{01} \mathrm{~B}_{02} \mathrm{~B}_{03}$ will be replaced by $R_{01} R_{02} R_{03} R_{01}^{\dagger} B_{01} R_{02}^{\dagger} B_{02} R_{03}^{\dagger} B_{03}=$ $\mathrm{R}_{01} \mathrm{R}_{02} \mathrm{R}_{03} \mathrm{G}_{01} \mathrm{G}_{02} \mathrm{G}_{03}$. This is how we construct an $\mathrm{im}$ proved Fourier transform algorithm (IQFT). For IQFT we need one additional type of gates, instead of diagonal B-gates, we use nondiagonal $\mathrm{R}$ and $\mathrm{G}$. To illustrate the obvious general procedure we write out the whole IQFT algorithm for $n=4$ qubits (compare with (8))

$$
\begin{aligned}
U_{\text {IQFT }}= & T_{03} T_{12} A_{0} R_{01} R_{02} R_{03} G_{01} G_{02} G_{03} A_{1} \\
& R_{12} R_{13} G_{12} G_{13} A_{2} R_{23} G_{23} A_{3} .
\end{aligned}
$$

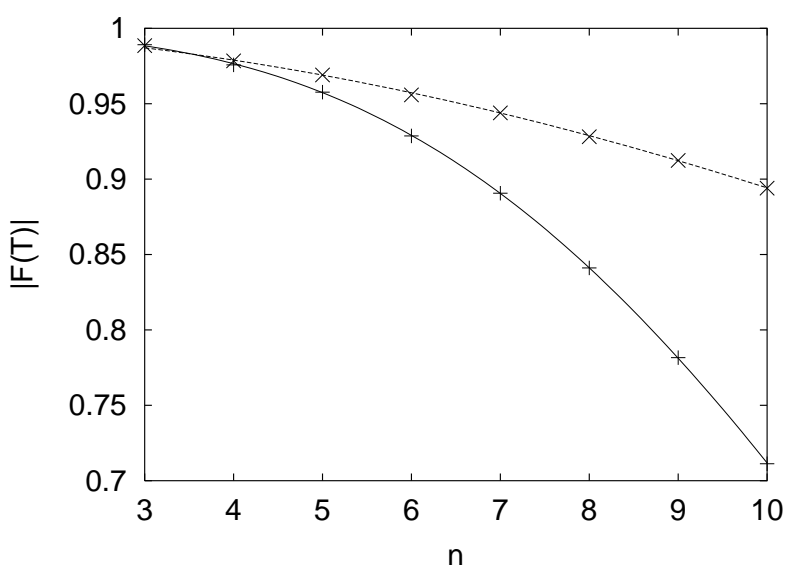

FIG. 2. Dependence of fidelity $|\langle F(T)\rangle|$ on the number of qubits $n$ for QFT (pluses) and IQFT algorithms (crosses), for fixed $\delta=0.04$. Numerical averaging over 50 GUE realizations is performed. Full curve is $\exp \left(-\delta^{2}\left\{0.236 n^{3}-0.38 n^{2}+1.45 n\right\}\right)$ and dashed is $\exp \left(-\delta^{2}\left\{0.61 n^{2}+0.89 n\right\}\right)$. For $n=10$ the trace is approximated by an average over 200 gaussian random register states.

Such IQFT algorithm consists of a total $T=[n(2 n+$ $1) / 2$ ] gates (note that it does not pay of to replace block with a single $\mathrm{B}$ gate as we have done, so we could safely leave $\mathrm{B}_{23} \equiv \mathrm{R}_{23} \mathrm{G}_{23}$ ). The correlation function for IQFT algorithm is shown in bottom fig.1. Almost all off-diagonal correlations are greatly reduced (to the level $\propto 1 / \mathcal{N}^{2}$ ), leaving us only with a dominant diagonal. If we had only diagonal elements, the fidelity would 
be $\langle F(T)\rangle=1-\delta^{2} \frac{T}{2}$, (as in the case of noisy perturbation or decoherence, however, with a different physical meaning of the strength scale $\delta$ ) where the number of gates scales as $T \propto n^{2}$. From the pictures (11) it is clear that we have very fast correlation decay for IQFT so that the correlation sum $\chi$ has decreased from $\chi \propto n^{3}$ to $\chi \propto n^{2}$ behavior. To further illustrate this, we have numerically calculated the fidelity by simulating QA and applying perturbation $\exp (-i \delta V)$ at each gate. The results are shown in fig.2. Difference between $\propto n^{2}$ and $\propto n^{3}$ behavior is nicely seen. As we have argued before, the sum of 2point correlator (5) gives us only the first nontrivial order in $\delta$-expansion. For dynamical systems, being either integrable or mixing and ergodic, it has been shown 11] that also higher orders of (14) can typically be written as simple powers of the correlation sum $\chi$, so that the fidelity has a simple functional form $F(t)=\exp \left(-\chi \delta^{2}\right)$. Although QA has quite inhomogeneous time-dependence, we may still hope that $\exp \left(-\chi \delta^{2}\right)$ is a reasonable approximation to the fidelity also at higher orders in $\delta$. This is in fact the case as can be seen in fig. 3. Note also that the leading coefficient in the exponent for IQFT, $\lim _{n \rightarrow \infty} \chi / n^{2}=0.61$, is close to the theoretical minimum of 0.5 .

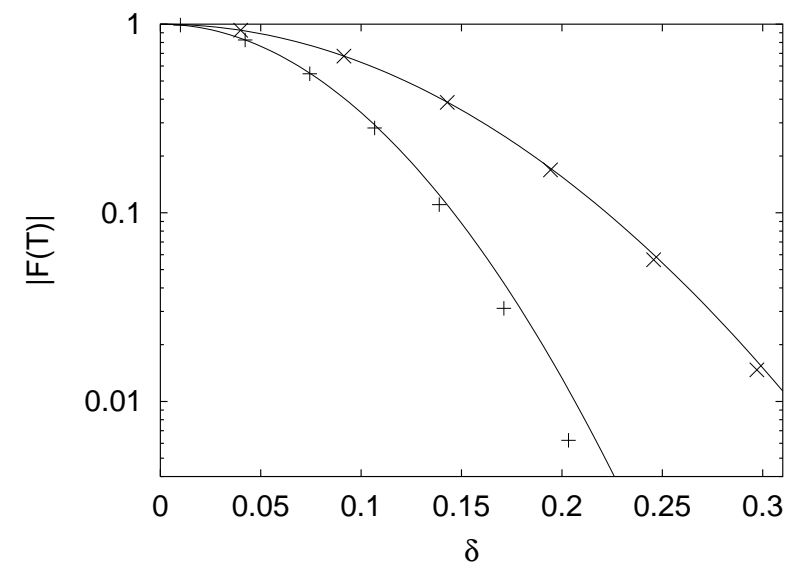

FIG. 3. Dependence of fidelity $|\langle F(T)\rangle|$ on $\delta$ for QFT (pluses) and IQFT (crosses), for fixed $n=8$. Solid curves are functions $\exp \left(-\chi \delta^{2}\right)$ (see text) with $\chi$ calculated analytically (9) and equal to $\chi=108$ for QFT and 46.6 for IQFT.

As the definition of what is a fundamental single gate is somehow arbitrary, the problem of minimizing the sum $\chi$ depends on a given technical realization of gates and the nature of the residual perturbation $V$ for an experimental setup. We should mention that the optimization becomes harder if we consider few-body (e.g. two-body random) perturbation. This is connected with the fact that quantum gates are two-body operators and can perform only a very limited set of rotations on a full Hilbert space and consequently have a limited capability of reducing correlation functions in a single step. However, our simple approach based on $n$-body random matrices seems reasonable if errors due to unwanted few-body qubit interactions can be eliminated by other methods [5.6].
In conclusion, we have presented a novel approach to the stability of time-dependent quantum dynamics applied to the fidelity of quantum computation. For an uncorrelated time-dependent perturbation, the decay of fidelity does not depend on dynamics, however, for a static perturbation characterizing faulty gates the system is more stable, as reflected in a higher fidelity, the more "chaotic" it is and the faster correlations decay it has. Our idea is demonstrated on example of QFT algorithm perturbed by a GUE matrix, devising an alternative QFT which is qualitatively more robust against a static random perturbation of the gates. It is an interesting question how this dynamical enhancement of stability relates to "chaotic melting" of a static quantum computer 13].

Discussions with T. H. Seligman are gratefully acknowledged. The work has been supported by the ministry of Education, Science and Sport of Slovenia.

[1] R. P. Feynman, Found. Phys. 16, 507 (1986).

[2] M. A. Nielsen and I. L. Chuang, Quantum Computation and Quantum Information (Cambridge UP, Cambdridge 2001); A. Steane, Rep. Prog. Phys. 61, 117 (1998); A. Ekert and R. Jozsa, Rev. Mod. Phys. 68, 733 (1996); D. P. DiVincenzo, Science 270, 255 (1995).

[3] P. W. Shor, in Proceedings of the 35th Annual Symposium on Fundations of Computer Science, ed. S. Goldwasser (IEEE Computer Society, Los Alamitos, 1994); D. Coppersmith, IBM research report RC19642 (1994); D. Deutsch, unpublished (1994).

[4] I. L. Chuang et al, Science 270, 1633 (1995).

[5] A. Steane, Proc. R. Soc. London A 452, 2551 (1996); A. R. Calderbank and P. W. Shor, Phys. Rev. A 54, 1098 (1996).

[6] L. Tian and S. Lloyd, Phys. Rev. A 62, R50301 (2000).

[7] J. Gea-Banacloche, Phys. Rev. A 57, R1 (1998); ibid. 60, 185 (1999); ibid. 62, 62313 (2000).

[8] P. H. Song and D. L. Shepelyansky, Phys. Rev. Lett. 86, 2162 (2001); B. Georgeot and D. L. Shepelyansky, preprints quant-ph/0101004 and quant-ph/0102082

[9] C. Miguel, J. P. Paz and R. Perazzo, Phys. Rev. A 54 2606 (1996); C. Miguel, J. P. Paz and W. H. Zurek, Phys. Rev. Lett. 783971 (1997); J. I. Cirac and P. Zoller, Phys. Rev. Lett. 74, 4091 (1995).

[10] A. Peres, Quantum Theory: Concepts and Methods (Kluwer AP, Dordrecht 1995), p366.

[11] T. Prosen, preprint quant-ph/0106149.

[12] Y. S. Weinstein et al, Phys. Rev. Lett. 861889 (2001); L. M. K. Vandersypen et al, ibid. 855452 (2000).

[13] G. Benenti, G. Casati and D. L. Shepelyansky, preprint quant-ph/0009084. 